March 1999

ADP-99-14/T357

\title{
Charm in the Nucleon
}

\author{
F.M. Steffens \\ Instituto de Fisica - USP, C.P. 66 318, 05315-970, SP, Brazil \\ W. Melnitchouk \\ Institut für Kernphysik, Forschungszentrum Jülich, D-52425 Jülich, Germany \\ A.W. Thomas \\ Department of Physics and Mathematical Physics, and Special Research Center for the Subatomic \\ Structure of Matter, University of Adelaide, 5005, Australia
}

\begin{abstract}
A next-to-leading order analysis of inelastic electroproduction of charm is performed using an interpolating scheme which maps smoothly onto massless QCD evolution at large $Q^{2}$ and photon-gluon fusion at small $Q^{2}$. In contrast with earlier analyses, this scheme allows the inclusion of quark and target mass effects and heavy quark thresholds, as well as possible non-perturbative, or intrinsic, charm contributions. We find no conclusive evidence in favor of an intrinsic charm component in the nucleon, although several data points which disagree with perturbative QCD expectations will need to be checked by future experiments.
\end{abstract}

\section{INTRODUCTION}

Understanding the role played in the nucleon by heavy quarks, such as the charm quark, is necessary for a number of reasons. Firstly, one cannot claim to have unraveled the rich structure of the nucleon sea until one has mapped out the details of the distribution of its virtual charm and heavier" flavors. Secondly, in the absence of a direct probe of gluons, charm leptoproduction remains one of the main sources of information on the nucleon's gluon distribution. Furthermore, tagging charm in neutrino and antineutrino scattering allows one to probe the strange and antistrange quark densities in the nucleon. From a more theoretical

\footnotetext{
${ }^{1}$ Although in practice the direct accessibility of bottom and top quark densities is likely to remain elusive for some time.
} 
point of view, in order to have a reliable procedure through which to analyze deep-inelastic scattering data, one needs to consistently incorporate heavy quark masses and threshold effects in the QCD evolution equations.

Recently there have been important new data on the charm structure function, $F_{2}^{c}$, of the proton from the H1 [1] and ZEUS [2] Collaborations at HERA, which have probed the small- $x$ region down to $x=8 \times 10^{-4}$ and $2 \times 10^{-4}$, respectively. At these values of $x$, the charm contribution to the total proton structure function, $F_{2}^{p}$, is found to be around $25 \%$, which is a considerably larger fraction than that found by the European Muon Collaboration at CERN [3] at somewhat larger $x$, where it was only $\sim 1 \%$ of $F_{2}^{p}$. Extensive theoretical analyses in recent years have generally served to confirm that the bulk of the $F_{2}^{c}$ data can be described through perturbative generation of charm within QCD.

At the same time, there are several lingering pieces of data which seem to suggest the possibility that a small component of charm exists which is intrinsically non-perturbative in origin [4 8]. One of these is the EMC data [3] at large $x$, some of which appear to lie above the perturbative QCD predictions, and which have in the past been taken [4] as evidence in favor of a non-perturbative, or "intrinsic" charm component. Furthermore, as recently discussed in Ref. [G], there are some indications of intrinsic charm also from hadronic reactions, such as leading charm production in $\pi N$ and $Y N$ scattering. As found in Ref. [9], some intrinsic charm may account for the larger than expected number of fast correlated $J / \psi$ pairs seen in the NA3 $\pi N$ experiment at CERN [10], as well as for the anomalous polarization of $J / \psi$ seen in inclusive $J / \psi$ production in $\pi N$ collisions [11].

The initial analyses of the leptoproduction data suggested an intrinsic charm component of the nucleon with normalization of around $1 \%$, although subsequent, more sophisticated, treatments incorporating higher-order effects tended to find only a fraction of a percent. The basic starting assumption in these analyses is that the perturbative generation of charm proceeds through the photon-gluon fusion process, illustrated in Figure 1. In their pioneering analysis, Hoffman and Moore [5] considered the $\mathcal{O}\left(\alpha_{s}\right)$ corrections to the intrinsic charm distributions, as well as quark and target mass effects on the charm cross section. With these refinements, their work suggested that an intrinsic charm component of the proton, at a level of order $0.3 \%$, was allowed - even required - by the data. In a more recent study, Harris, Smith and Vogt [6] reanalyzed the EMC data within the framework of Ref. [5] with updated parton distributions, and $\mathcal{O}\left(\alpha_{s}\right)$ corrections to the hard scattering cross section. They found that while no clear statements about intrinsic charm could be made at lower energy transfers, $\nu \lesssim 100 \mathrm{GeV}$, data at larger $\nu \sim 170 \mathrm{GeV}$ could best be fitted with an additional $0.86 \pm 0.60 \%$ intrinsic charm component [6].

Despite these claims of evidence for intrinsic charm, a number of recent global parameterizations of parton distributions 12,13 have concluded that all $F_{2}^{c}$ data, including those from the EMC, can be understood in terms of perturbative QCD alone, with no additional intrinsic charm component necessary. In view of these conflicting results, it seems important that the question of the presence or otherwise of intrinsic charm be addressed using the latest available theoretical techniques for calculating $F_{2}^{c}$, as well as different models for intrinsic charm.

To set the context for our analysis, we should point out a number of features which have been common to all earlier treatments of intrinsic charm in leptoproduction. Firstly, one invariably assumes that photon-gluon fusion (together with perturbative QCD corrections) 
is solely responsible for perturbatively generated charm, irrespective of the scale $Q^{2}$ at which one works. In light of recent developments in incorporating quark mass effects in perturbative QCD, it is now in principle possible to go beyond this approximation and include more appropriate treatments in kinematic regions where photon-gluon fusion alone may not be the only relevant perturbative mechanism. A second point concerns the way that intrinsic charm is combined with the perturbative contributions. In particular, no treatment to date has considered the effects of $Q^{2}$ evolution of the non-perturbative charm distribution from the "intrinsic" scale to the scale where data are taken.

In this paper we perform a comprehensive next-to-leading order analysis of the charm production data in which we compare several different methods of implementing charm in QCD structure functions. In particular, we consider:

- Massless Evolution. This approach assumes that below a certain scale, $\mu^{2}$, there are $n_{f}$ active flavors. Above this scale, one switches the number of flavors to $n_{f}+1$ in the coupling constant, coefficient functions and in the parton distributions. This scheme is sometimes referred to as the Variable Flavor Number Scheme (VFNS).

- Photon-Gluon Fusion. In this scheme, the heavy quark is never treated as a parton, in the sense that the number of active flavors does not change, and a quark distribution for the heavy quark is never introduced. The entire contribution to the physical structure function from heavy quarks is generated through the photon-gluon fusion process depicted in Figure 1. This scheme is usually referred to as the Fixed Flavor Number Scheme (FFNS).

- Interpolating Schemes. While photon-gluon fusion should provide a reliable description of charm production in the region close to $m_{c}^{2}$, it certainly should fail at large scales because of the lack of resummation of the logarithms in $m_{c}^{2}$. Conversely, massless evolution should be good at large $Q^{2}$, but not in the region around $m_{c}^{2}$. Schemes such as that proposed by Aivazis et al. [14] reproduce the relevant features of both the VFNS and FFNS approaches - namely, a reduction to FFNS when $Q^{2} \sim m_{c}^{2}$, and to VFNS when $Q^{2} \gg m_{c}^{2}$. Unlike Ref. [14], however, we include the full quark mass dependence not only in the gluon sector, but also in the quark sector, which has become possible since the recent work of Kretzer and Schienbein 15.

Throughout the analysis we shall work in the $\overline{\mathrm{MS}}$ scheme, and introduce an explicit charm distribution at a scale $m_{c}^{2}$ for the VFNS and interpolating schemes. Furthermore, our philosophy is that if there is any intrinsic charm at all in the nucleon, it should be considered as a parton distribution for all scales $Q^{2} \geq m_{c}^{2}$, if one is to use $\overline{\mathrm{MS}}$ anomalous dimensions when evolving the distributions.

In the following sections we describe in turn the three schemes for incorporating charm, beginning with the standard massless QCD evolution and the photon-gluon fusion process in Sections II and III, respectively. The interpolating scheme connecting these two limiting cases is described in Section IV. Incorporation of intrinsic charm into the analysis is outlined in Section V, where we survey several models for the non-perturbative charm component. Finally, in Section VI some conclusions are drawn from the analysis. 


\section{MASSLESS EVOLUTION (VFNS)}

The simplest approach to describing charm in the nucleon is to assume that the charm distribution is $c\left(x, \mu^{2}\right)=\bar{c}\left(x, \mu^{2}\right)=0$ below a certain scale, $\mu$, and evolve charm quarks as massless partons. Indeed, until recently, this has been the standard approach adopted in global analyses of parton distribution functions. In a scheme such as $\overline{\mathrm{MS}}$, the scale $\mu$ is taken to be the charm quark mass, $m_{c}$ [16]. Above threshold, $Q^{2}>m_{c}^{2}$, the number of active flavors increases by one in the coupling constant, splitting and coefficient functions. In this way the charm quark distribution is generated solely through radiative corrections from gluons and light quarks present at $Q^{2}<m_{c}^{2}$.

At next-to-leading order, the charm quark contribution to the proton structure function is given by:

$$
F_{2}^{c(\mathrm{VFNS})}\left(x, Q^{2}\right)=e_{c}^{2} C_{q}\left(x, Q^{2}\right) \otimes\left(x c\left(x, Q^{2}\right)+x \bar{c}\left(x, Q^{2}\right)\right)+e_{c}^{2} C_{g}\left(x, Q^{2}\right) \otimes x g\left(x, Q^{2}\right)
$$

with the boundary condition $c\left(x, m_{c}^{2}\right)=\bar{c}\left(x, m_{c}^{2}\right)=0$. The convolution " $\otimes$ " here is defined by $f(x) \otimes h(x) \equiv \int_{x}^{1} d y f(x / y) h(y)$. The quark and gluon coefficient functions are those for massless quarks:

$$
\begin{aligned}
C_{q}\left(x, Q^{2}\right)= & \delta(1-x)+\frac{\alpha_{s}\left(Q^{2}\right)}{4 \pi} C_{F}\left\{4\left(\frac{\ln (1-x)}{1-x}\right)_{+}-\frac{3}{(1-x)_{+}}-2(1+x) \ln (1-x)\right. \\
& \left.-2 \frac{1+x^{2}}{1-x} \ln x+6+4 x\right\}, \\
C_{g}\left(x, Q^{2}\right)= & \frac{\alpha_{s}\left(Q^{2}\right)}{4 \pi} 4 T_{F}\left\{\left(1-2 x+2 x^{2}\right) \ln \frac{1-x}{x}-4+32 x(1-x)\right\},
\end{aligned}
$$

where the $(1-x)_{+}$refers to the standard "+" prescription [17].

The gluon coefficient function, $C_{g}$, is a pure singlet quantity, and as such is proportional to the number of active flavors. Since one is calculating the contribution to $F_{2}^{p}$ from a single flavor, $C_{g}$ defined in Eq.(2b) is the usual gluon coefficient function divided by the number of active flavors. The gluon distribution is evolved by taking as input a gluon distribution function at a scale $m_{c}^{2}$, together with a quark singlet distribution, $\Sigma\left(x, m_{c}^{2}\right)=$ $\sum_{q=u, d, s, c}\left(q\left(x, m_{c}^{2}\right)+\bar{q}\left(x, m_{c}^{2}\right)\right)$. Using the boundary condition $c\left(x, m_{c}^{2}\right)=\bar{c}\left(x, m_{c}^{2}\right)=0$, the evolution is performed with 4 flavors.

The evolution of the charm quark density is slightly more involved. Firstly, one evolves the singlet combination from $m_{c}^{2}$ to $Q^{2}$ using the full number of active flavors. The quark non-singlet combination, $\eta\left(x, Q^{2}\right)=\sum_{q=u, d, s}\left(q\left(x, Q^{2}\right)+\bar{q}\left(x, Q^{2}\right)\right)-3\left(c\left(x, Q^{2}\right)+\bar{c}\left(x, Q^{2}\right)\right)$, is then evolved over the same range. Since $c\left(x, m_{c}^{2}\right)=\bar{c}\left(x, m_{c}^{2}\right)=0$, it turns out that one is in practice evolving $\sum_{q=u, d, s}\left(q\left(x, m_{c}^{2}\right)+\bar{q}\left(x, m_{c}^{2}\right)\right)$ first as a singlet and then as a non-singlet. The charm distribution at $Q^{2}$ is then given by:

$$
c\left(x, Q^{2}\right)+\bar{c}\left(x, Q^{2}\right)=\frac{1}{4}\left(\Sigma\left(x, Q^{2}\right)-\eta\left(x, Q^{2}\right)\right) .
$$

2 Just as for the $c$ quark, the $u, d$, and $s$ quarks are light, so too for heavier quarks, such as $b$, the $u, d, s$ and $c$ quarks would be light, and so on. 
For large $Q^{2}, Q^{2} \gg m_{c}^{2}$, one expects the generation of charm through massless evolution to be a good approximation, a result confirmed in previous studies [18]. However, at smaller $Q^{2}, Q^{2} \sim m_{c}^{2}$, mass effects become important, and this approach eventually breaks down. In this region a better approximation is the photon-gluon fusion process, which we discuss next.

\section{PHOTON-GLUON FUSION (FFNS)}

In the photon-gluon fusion approach the charm quark is treated not as a parton, but rather as a heavy quark, so that a charm quark distribution is never explicitly introduced. Hence, $c=\bar{c}=0$ for all $x$ and $Q^{2}$. The charm structure function is given directly by a convolution of the gluon distribution and the hard photon-gluon cross section, Figure 1.

At lowest order, the only process producing a heavy $q \bar{q}$ pair is the photon-gluon fusion, and the structure function is given by:

$$
F_{2}^{c(\mathrm{FFNS})}\left(x, Q^{2}\right)=e_{c}^{2} \frac{\alpha_{s}\left(\mu^{2}\right)}{4 \pi} \int_{x}^{z_{\max }} d z H_{g}\left(z, Q^{2}, m_{c}^{2}\right) \frac{x}{z} g\left(\frac{x}{z}, \mu^{2}\right),
$$

where $z_{\max }=1 /\left(1+4 m_{c}^{2} / Q^{2}\right)$ and $\mu^{2}$ is the mass factorization (and renormalization) scale. The partonic cross section for producing a massive quark pair is given by:

$$
\begin{aligned}
H_{g}\left(z, Q^{2}, m_{c}^{2}\right) & =4\left(1-2 z+2 z^{2}+4 z(1-3 z) \frac{m_{c}^{2}}{Q^{2}}-8 z^{2} \frac{m_{c}^{4}}{Q^{4}}\right) \ln \left(\frac{1+\beta}{1-\beta}\right) \\
& +\beta\left(32 z(1-z)-4-16 z(1-z) \frac{m_{c}^{2}}{Q^{2}}\right),
\end{aligned}
$$

with

$$
\beta=\sqrt{1-\frac{4 z}{(1-z)} \frac{m_{c}^{2}}{Q^{2}}} .
$$

Note that the coupling constant is calculated at $\mu^{2}$ with 3 light flavors, the same number as used throughout in $H_{g}$. There is no evolution equation in this approach - the distribution $g\left(x, \mu^{2}\right)$ is never evolved. Evolution equations are introduced only when the logs in $Q^{2} / m_{c}^{2}$ are resummed via the renormalization group equations. In that case a heavy quark distribution is introduced and one recovers Eq.(11).

One should mention that the gluon distribution in Eq.(1) is that given by a leadingorder analysis of the data. This is because in Eq.(4) one has terms of the form $\alpha_{s}\left(\mu^{2}\right) \ln \left(Q^{2} / m_{c}^{2}\right) g\left(x, \mu^{2}\right)$, which are typical next-to-leading order contributions if the gluon distribution is calculated in leading order - hence one is calculating $\mathcal{O}\left(\alpha_{s}\right)$ corrections to the structure function. The use of a next-to-leading order gluon would produce next-to-nextto-leading order corrections, which would then require calculation of radiative corrections to the photon-gluon fusion term, and the addition of extra terms in Eq.(44) arising from heavy pair creation from light quarks [19].

The advantage of the photon-gluon fusion formulation is its simplicity. However, the use of Eq.(四) could be problematic at very large $Q^{2}$ because of the large $\ln \left(Q^{2} / m_{c}^{2}\right)$ term 
appearing in $H_{g}$. One way of circumventing this difficulty is to construct a scheme which retains the features of photon-gluon fusion at moderate $Q^{2}$, but maps onto massless evolution when $Q^{2}$ becomes very large.

\section{INTERPOLATING SCHEME}

The description of charm production from the nucleon at an arbitrary scale requires a scheme which consistently interpolates between the two limits of VFNS and FFNS. A number of authors have pursued the construction of such schemes, both for unpolarized |14, 18, 20,21] and polarized [22] scattering.

With the exception of Ref. [20], where the splitting functions were modified for quark mass effects, the essence of the interpolating schemes is to incorporate a mass dependence, as a function of $m_{c}^{2} / Q^{2}$, in the coefficient functions which would enable Eq.(1) to be recovered when $Q^{2} \gg m_{c}^{2}$, and Eq.(壮) when $Q^{2} \sim m_{c}^{2}$. By keeping the evolution equations unmodified, one ensures that the parton distributions (for the 3 light quarks, for the gluon, and for the newly introduced charm distribution) are defined consistently in the $\overline{\mathrm{MS}}$ scheme.

Until recently, quark mass corrections were only included in the gluon coefficient function, $H_{g}$, while the quark coefficient function was taken to have the form derived for massless quarks [12,14]. In fact, it is quite natural to think that the gluon sector, through the $P_{c g}$ splitting function, would be responsible for the generation and evolution of a heavy quark distribution. Furthermore, any quark mass correction (other than that appearing in the new, massive, scaling variable) would appear in the $\mathcal{O}\left(\alpha_{s}\right)$ coefficient functions only. In Ref. [15], Kretzer and Schienbein also calculated the quark mass corrections to the quark coefficient function. They found that these were small in absolute terms, but of a similar order of magnitude as the total $F_{2}^{c}$ in some regions of $x$, and for some choices of factorization scale. There are at least two reasons, therefore, why one needs to include these effects in the present analysis. Firstly, with the introduction of intrinsic charm the quark sector may become relatively more important than the gluon sector in the region of $x$ where intrinsic charm is non-zero; and secondly, the analysis of Ref. 15] focused on quark mass corrections to the quark coefficient functions at relatively small values of $x$, while here we also need to examine the size of these corrections at large $x$.

Of course, the full massive coefficient functions introduce extra mass logarithms in the quark and gluon sectors, resulting in double counting when using the evolution equations for the charm and gluon densities. This is reminiscent of a scenario where logarithms have not yet been resummed by the renormalization group equations. The problem can be circumvented easily enough by requiring that in the limit $Q^{2} \rightarrow \infty$ the massless coefficient functions are recovered after introducing subtraction terms $H_{q, g}^{\text {sub }}$ :

$$
\begin{aligned}
& \lim _{Q^{2} \rightarrow \infty}\left(H_{q}\left(z, Q^{2}, m_{c}^{2}\right)-H_{q}^{\mathrm{sub}}\left(z, Q^{2}, m_{c}^{2}\right)\right)=C_{q}\left(z, Q^{2}\right), \\
& \lim _{Q^{2} \rightarrow \infty}\left(H_{g}\left(z, Q^{2}, m_{c}^{2}\right)-H_{g}^{\mathrm{sub}}\left(z, Q^{2}, m_{c}^{2}\right)\right)=C_{g}\left(z, Q^{2}\right),
\end{aligned}
$$

where the factorization scale is chosen to be $\mu^{2}=Q^{2}$ for both the interpolating scheme and the VFNS (a choice to which we restrict ourselves throughout this analysis). The function 
$H_{q}\left(z, Q^{2}, m_{c}^{2}\right)$ in Eq.(7a) is the full massive quark coefficient function at $\mathcal{O}\left(\alpha_{s}\right)$, calculated in Ref. 15. The subtraction terms are given by:

$$
\begin{aligned}
& H_{q}^{\mathrm{sub}}\left(z, Q^{2}, m_{c}^{2}\right)=\frac{\alpha_{s}\left(Q^{2}\right)}{2 \pi} C_{F}\left[\frac{1+z^{2}}{1-z}\left(\ln \frac{Q^{2}}{m_{c}^{2}}-1-2 \ln (1-z)\right)\right]_{+}, \\
& H_{g}^{\mathrm{sub}}\left(z, Q^{2}, m_{c}^{2}\right)=\frac{\alpha_{s}\left(Q^{2}\right)}{\pi} T_{F} \ln \frac{Q^{2}}{m_{c}^{2}}\left(1-2 z+2 z^{2}\right) .
\end{aligned}
$$

Finally, one has:

$$
\begin{aligned}
F_{2}^{c}\left(x, Q^{2}\right) & =e_{c}^{2} \int_{\xi}^{1} d z\left(H_{q}\left(z, Q^{2}, m_{c}^{2}\right)-H_{q}^{\mathrm{sub}}\left(z, Q^{2}, m_{c}^{2}\right)\right) \frac{\xi}{z}\left(c\left(\xi / z, Q^{2}\right)+\bar{c}\left(\xi / z, Q^{2}\right)\right) \\
& +e_{c}^{2} \int_{x}^{z_{\max }} d z H_{g}\left(z, Q^{2}, m_{c}^{2}\right) \frac{x}{z} g\left(x / z, Q^{2}\right) \\
& -e_{c}^{2} \int_{\xi}^{1} d z H_{g}^{\mathrm{sub}}\left(z, Q^{2}, m_{c}^{2}\right) \frac{\xi}{z} g\left(\xi / z, Q^{2}\right),
\end{aligned}
$$

where the scaling variable $\xi$ includes quark mass corrections,

$$
\xi=\frac{1}{2} x\left(1+\sqrt{1+\frac{4 m_{c}^{2}}{Q^{2}}}\right) .
$$

The charm and gluon distributions in Eq.(9) are determined from evolution in exactly the same way as in the massless case described in Section II.

Figure 2 shows $F_{2}^{c}$ evaluated according to the three schemes described above at $x=0.05$ and 0.2 , for a $Q^{2}$ range relevant to the EMC data. In addition, for the interpolating scheme we also show the effect of neglecting the quark mass dependence in the quark coefficient function, so that $H_{q}-H_{q}^{\text {sub }}$ is replaced by $C_{q}$ in Eq.(9). For the parton distributions the GRV parameterizations [13] are used. Note, however, that in the GRV fit a charm quark distribution is never introduced explicitly, rather $F_{2}^{c}$ is always calculated via the FFNS. The charm density is generated from the GRV distributions by evolving with 3 flavors from $\mu^{2}=0.4 \mathrm{GeV}^{2}$ to $m_{c}^{2}$ in next-to-leading order, then from $m_{c}^{2}$ to $Q^{2}$ with 4 flavors according to the VFNS. For the FFNS calculation, the gluon distribution is evolved in leading order from $\mu^{2}=0.26 \mathrm{GeV}^{2}$ to $m_{c}^{2}$ with 3 flavors. (Note that the choice of $\mu^{2}$ in Figure 2 is only for the purpose of comparison with the other methods, which introduce charm at $m_{c}^{2}$. In the final calculations the scale $\mu^{2}=4 m_{c}^{2}$ will be used for the FFNS, which is also the value used in Ref. [13.)

At small $x$ the effect of the mass-corrected quark coefficient functions on $F_{2}^{c}$ turns out to be negligible, and only slight at larger $x$. As $Q^{2}$ becomes large, one can see in Figure 2 how the VFNS and interpolating schemes converge. Even at small $Q^{2}$ the difference between these is not large. On the other hand, while the FFNS provides a good approximation to the interpolating scheme for $Q^{2} \lesssim 30 \mathrm{GeV}^{2}$, it dramatically overestimates the full result at larger $Q^{2}$, especially at large $x$. Since this is the region where most of the relevant EMC data which we analyse lie, clearly we need to use the full interpolating scheme in order to draw reliable conclusions from our analysis.

Another source of uncertainty in the calculation of $F_{2}^{c}$ comes from the gluon distribution at large $x$, which at present is not very well constrained. To cover the full range of allowed 
gluon distributions, we use the maximum and minimum gluon distributions from the MRST [12] parameterizations in addition to that of GRV [13]. The resulting $F_{2}^{c}$ for the different next-to-leading order glue is shown in Figure 3, where evolution was again performed as described in the massless evolution section The data at small $x$ in Figure 3 are taken from the ZEUS Collaboration [2], while the large- $x$ data are from the earlier EMC experiment [3]. At small $x$ all of the parameterizations fit the data very well. At large $x$ the maximum-gluon MRST and GRV fits also provide good descriptions of the data, with the exception of the two points at $Q^{2}=60 \mathrm{GeV}^{2}$. The last data point at $x=0.44$ is not shown in the fits of Refs. [12,[3], even though this point appears to confirm the trend indicated by the $x=0.24$ point to lie somewhat above the perturbative QCD calculation. Neglecting the large- $x, Q^{2}=60$ $\mathrm{GeV}^{2}$ points, one would conclude that perturbative QCD fits the $F_{2}^{c}$ data very well, without any need for additional non-perturbative contributions [12,13]. On the other hand, taking these points seriously has led several authors [5,6] to conclude that the large- $x$ EMC data provide evidence for an intrinsic charm component of the nucleon.

In the next Section we shall study the large- $x$ EMC data more carefully, with the aim of ascertaining whether these can be understood perturbatively, or whether they can indeed be interpreted as suggesting that a perturbative QCD treatment alone is incomplete.

\section{NON-PERTURBATIVE CHARM}

The apparent discrepancy between some of the large- $x F_{2}^{c}$ data and predictions based solely on perturbative QCD has prompted several authors [- 6 [ to take seriously the possibility that an additional, non-perturbative, component of $F_{2}^{c}$ may be necessary to account for the data over the full range of $x$ and $Q^{2}$. In this Section we discuss how non-perturbative charm may affect $F_{2}^{c}$, particularly at large $x$, and how the intrinsic contributions can be included on the same footing as the perturbative effects.

In earlier analyses [5, 6] intrinsic charm distributions have simply been added to the perturbatively generated $F_{2}^{c}$,

$$
F_{2}^{c}\left(x, Q^{2}\right)=F_{2}^{c(\text { pert })}\left(x, Q^{2}\right)+F_{2}^{c(\mathrm{IC})}\left(x, Q^{2}\right),
$$

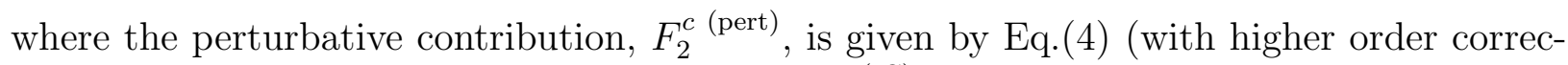
tions), while the intrinsic charm contribution, $F_{2}^{c}$ (IC) , in its simplest form is:

$$
F_{2}^{c(\mathrm{IC})}=e_{c}^{2} x\left(c^{\mathrm{IC}}+\bar{c}^{\mathrm{IC}}\right) .
$$

In practice, $\mathcal{O}\left(\alpha_{s}\right)$ contributions to Eq.(12) are fully implemented in this analysis (the relevant expressions are given in Ref. [5]).

Within the interpolating scheme of Section IV, the most natural way to implement intrinsic charm in $F_{2}^{c}$ is to modify the boundary condition for the charm quark distribution. Instead of $c\left(x, \mu^{2}\right)=\bar{c}\left(x, \mu^{2}\right)=0$, one now has non-zero distributions at the scale $\mu^{2}=m_{c}^{2}$. The physical reasoning for this is that if there are non-perturbative processes producing charm in the nucleon, this charm can be resolved (brought on its mass shell) only when the system has sufficient energy. In the $\overline{\mathrm{MS}}$ scheme, the scale at which the number of flavors changes from 3 to 4 is $\mu^{2}=m_{c}^{2}$, so that regardless of the dynamical origin of the charm, there 
will be enough energy in the system to open a new active flavor channel for $Q^{2}>m_{c}^{2}$. With this in mind, we next discuss several non-perturbative models which attempt to describe the generation of intrinsic charm in the nucleon.

\section{A. Five-Quark Component of the Nucleon (IC1)}

Based on the initial observation [23] that the charm production cross section in hadronic collisions was larger than that predicted in leading order perturbative QCD, Brodsky et

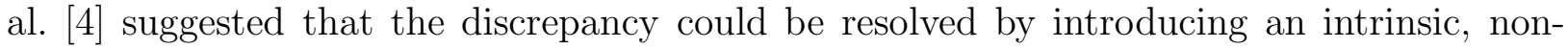
perturbative, charm component in the nucleon wave function. In this model, which we shall refer to as "IC1", the nucleon is assumed to contain, in addition to the lowest energy three-quark Fock state, a more complicated, five-quark configuration on the light-cone:

$$
|p\rangle=c_{0}|u u d\rangle+c_{1}|u u d c \bar{c}\rangle,
$$

where $c_{0}^{2}\left(c_{1}^{2}\right)$ is the three (five)-quark probability. In order to explain the original data [23], the normalization of the latter was chosen to be $1 \%$ [4]. Assuming the five-quark wave function to be inversely proportional to the light-cone energy difference between the nucleon ground state and the five-quark excited state, one finds that the $x$-dependence of the $c$ quark distribution is given by [4]:

$$
c^{\mathrm{IC} 1}(x)=6 x^{2}\left((1-x)\left(1+10 x+x^{2}\right)-6 x(1+x) \log 1 / x\right) .
$$

The anticharm distribution has the same shape as the charm distribution in this model, $\bar{c}^{\mathrm{IC} 1}(x)=c^{\mathrm{IC} 1}(x)$.

Because the intrinsic charm in this model is assumed to be generated through $g g \rightarrow$ $c \bar{c}$ processes, with each gluon originating from different valence quarks, the $c \bar{c}$ probability scales like $\alpha_{s}^{2}\left(m_{c}^{2}\right) / m_{c}^{2}$ relative to the perturbative component [6,24]. This contribution was therefore interpreted in Refs. 6,24] as a higher-twist effect. Our philosophy here is that since this is generated non-perturbatively, the resulting non-perturbative intrinsic charm distribution calculated at $m_{c}^{2}$ should be evolved as leading twist, on the same footing as the perturbative contribution. Since there is only one kind of charm quark, irrespective of its origin, QCD corrections should affect both the perturbative and non-perturbative distributions identically.

\section{B. Meson Cloud Model (IC2)}

An alternative to the five-quark intrinsic charm model was considered in Refs. 25 27, in which the charm sea was assumed to arise from the quantum fluctuation of the nucleon to a virtual $\bar{D}^{0}+\Lambda_{c}^{+}$configuration. In the following we shall refer to this model as "IC2". The nucleon charm radius [25] and the charm quark distribution 26] were both estimated in this framework. Furthermore, the effects of hard charm distributions on large- $x$ HERA cross sections, and in particular on the so-called HERA anomaly [28,29, were studied in Ref. [27].

The meson cloud model for the long-range structure of the nucleon has been used extensively to describe various flavor symmetry breaking phenomena observed in deep-inelastic 
scattering and related experiments. It offers a natural explanation of the $\bar{d}$ excess in the proton over $\bar{u}$ [30,31] in terms of a pion cloud, which itself is a necessary ingredient of the nucleon by chiral symmetry. It also provides an intuitive framework to study the strangeness content of the nucleon, through the presence of the kaon cloud [32]. Whether the same philosophy can be justified for a cloud of heavy charm mesons and baryons around the nucleon is rather more questionable given the large mass of the fluctuation. Nevertheless, to a crude approximation, one may take the meson cloud framework as indicative of the possible order of magnitude and shape of the non-perturbative charm distribution. Furthermore, a natural prediction of this model is that the $c$ and $\bar{c}$ distributions are not symmetric.

In the meson cloud model, the distribution of charm and anticharm quarks in the nucleon at some low hadronic scale can be approximated by [27]:

$$
\begin{aligned}
c^{\mathrm{IC} 2}(x) & \approx \frac{3}{2} f_{\Lambda_{c} / N}(3 x / 2), \\
\bar{c}^{\mathrm{IC} 2}(x) & \approx f_{\bar{D} / N}(x),
\end{aligned}
$$

where

$$
f_{\bar{D} / N}(x)=\frac{1}{16 \pi^{2}} \int_{0}^{\infty} d k_{\perp}^{2} \frac{g^{2}\left(k_{\perp}^{2}, x\right)}{x(1-x)\left(s-M^{2}\right)^{2}}\left(\frac{k_{\perp}^{2}+\left[M_{\Lambda_{c}}-(1-x) M\right]^{2}}{1-x}\right)
$$

is the light-cone distribution of $\bar{D}^{0}$ mesons in the nucleon, and $f_{\Lambda_{c} / N}(x)=f_{\bar{D} / N}(1-x)$ is the corresponding distribution of $\Lambda_{c}^{+}$baryons. In Eq.(16) the function $g$ describes the extended nature of the $\bar{D} \Lambda_{c} N$ vertex, with the momentum dependence parameterized by $g^{2}\left(k_{\perp}^{2}, x\right)=g_{0}^{2}\left(\Lambda^{2}+M^{2}\right) /\left(\Lambda^{2}+s\right)$, where $s$ is the $\bar{D} \Lambda_{c}$ center of mass energy squared and $g_{0}$ the $\bar{D} \Lambda_{c} N$ coupling constant at the pole, $s=M^{2}$. As a first approximation, one might take $g_{0}$ to be of the same order of magnitude as the $\pi N N$ coupling constant. In Ref. [33] this coupling constant was estimated within a QCD sum rule calculation.

\section{Intrinsic Charm Distributions}

The $c$ and $\bar{c}$ distributions in the intrinsic charm models IC1 and IC2 are shown in Figure $₫$, each normalized to a common value of $1 \%$. For the IC2 model this corresponds to a cutoff $\Lambda \approx 2.2 \mathrm{GeV}$ (for a probability of $0.5 \%$, for example, one would need $\Lambda \approx 1.7 \mathrm{GeV}$ ). Quite interestingly, the shapes of the $c$ quark distributions are quite similar in the two models, with $x c$ peaking at around $x \sim 0.3$. However, because the IC2 model gives a significantly harder $\bar{c}$ distribution, while IC1 implies that $c$ and $\bar{c}$ are equal, the resulting structure function, $F_{2}^{c}$, will be somewhat harder in the IC2 model. For comparison, a typical (soft) perturbatively-generated charm distribution is also shown in Figure 4, evaluated from the MRST parameterization [12] (with the maximal gluon) at $Q^{2}=5 \mathrm{GeV}^{2}$.

The effects of the modified boundary conditions incorporating non-zero intrinsic charm distributions are shown in Figure 5 for the GRV parameterization [13 and for 1\% intrinsic charm normalizations, at $Q^{2}=25,45$ and $60 \mathrm{GeV}^{2}$. The data at $60 \mathrm{GeV}^{2}$ are well fitted with a $1 \%$ IC1 component, although with the IC2 model one slightly overestimates the $x=0.44$ data point, due to its very hard $\bar{c}$ distribution. At lower $Q^{2}$ values, however, the addition of a $1 \%$ intrinsic charm component, from either model, overestimates the large- $x$ points. 
This finding is essentially independent of the parton distribution functions employed, as Figure 6 illustrates for the MRST distributions [12]. From this one can conclude that with a $1 \%$ intrinsic charm component one cannot simultaneously resolve the large- $x$ discrepancy for the large- $Q^{2}$ data, and maintain a satisfactory fit to the data at lower $Q^{2}$. One could imagine introducing a $Q^{2}$-dependent normalization for the intrinsic charm to improve the fits to the 25 and $45 \mathrm{GeV}^{2}$ data, although this solution would not be well grounded theoretically.

To compare with the procedure for incorporating intrinsic charm adopted in the earlier analysis in Ref. [5], we show in Figure 7 the $F_{2}^{c}$ obtained from the FFNS through Eq.(11), and the $\mathcal{O}\left(\alpha_{s}\right)$ corrections to the intrinsic charm component, $F_{2}^{c}$ (IC) - see Eq.(57) of Ref. [5]. (Note that although the FFNS curves appear to lie slightly below the data for $Q^{2}=25$ and $45 \mathrm{GeV}^{2}$, inclusion of $\mathcal{O}\left(\alpha_{s}^{2}\right)$ corrections leads to a slight improvement for the lower $Q^{2}$ data, without much effect on the data at $60 \mathrm{GeV}^{2}$ [13].) The results are qualitatively similar to those obtained from the full interpolating scheme, namely the data at different $Q^{2}$ seem to require different functional forms and normalizations for the intrinsic charm.

By varying the amount of intrinsic charm that the data can accommodate, one can attempt to improve the overall fit at all values of $Q^{2}$. Using the minimum gluon fit of the MRST parameterization one can determine the maximum intrinsic charm allowed by the data for both the IC1 and IC2 models. Slightly better fits can be obtained with a $0.75 \%$ IC1 charm distribution, and with a $0.4 \%$ IC2 distribution, as indicated in Figure 8 for $Q^{2}=45$ and $60 \mathrm{GeV}^{2}$. (The quality of the fit at $Q^{2}=25 \mathrm{GeV}^{2}$ is similar to that for the $45 \mathrm{GeV}^{2}$ data.) However, even given the different shapes of the charm and anticharm distributions in the IC1 and IC2 models, it is still quite difficult to obtain a quality fit to data at all $Q^{2}$ values. For the Hoffman and Moore procedure [5] with the FFNS, one finds similar difficulties in reconciling the $Q^{2}=60 \mathrm{GeV}^{2}$ data with those at lower $Q^{2}$, even as a function of the intrinsic charm model, and normalization (c.f. Figure 9), although there may be a slight preference for IC2 with $0.4 \%$ normalization.

In view of the difficulties in obtaining a simultaneous fit to the $F_{2}^{c}$ data at all measured values of $x$ and $Q^{2}$ with either the perturbative-only, or the intrinsic charm scenarios, it seems imperative that the data, particularly those at large $x$ and $Q^{2}$, be confirmed by independent measurements.

\section{CONCLUSION}

Unraveling the rich structure of the nucleon sea is an ongoing endeavor which has taught us a number of important and sometimes surprising lessons in recent years. Far from being a homogeneous background in which the valence quarks reside, the sea has proved to exhibit considerable intrinsic structure of its own. Perhaps the most conspicuous structure is that in the light quark sea of the proton, where a number of experiments have now confirmed beyond any doubt a significant excess of $\bar{d}$ over $\bar{u}$ quarks [30]. This in turn has created an environment in which the importance of non-perturbative effects in the nucleon is appreciated to a far greater extent, even when discussing structure at deep-inelastic energy scales [31].

More speculative, and less constrained experimentally, are suggestions that the proton sea for heavier flavors might also exhibit characteristics which cannot be attributed to perturbative QCD mechanisms alone [27.32]. A prime example would be the presence of asymmetric sea quark and antiquark distributions, which have been searched for in the strange sector in 
both deep-inelastic neutrino and antineutrino scattering, as well as in electromagnetic form factors at low energies [34]. Indeed, there is no symmetry principle in QCD which would prevent sea quarks and antiquarks having different momentum distributions, just as there is no symmetry requiring the $\bar{d}$ and $\bar{u}$ sea to be equivalent.

More challenging still is to identify possible non-perturbative effects in the charm sector, especially since the magnitude of the total charm component of non-charm hadrons is tiny. Nevertheless, a number of pioneering studies [4 6] of various charm production reactions have left us with lingering doubts as to whether perturbative QCD is the full story behind these processes. On the other hand, the evidence doesn't appear to be conclusive enough to warrant introduction of intrinsic charm distributions in global data parameterizations 12, 13, 35, which to date have generated the charm structure function purely perturbatively.

The present analysis has been an attempt to resolve the issue of whether the charm electroproduction data do indeed support the existence of non-perturbative components of the nucleon wave function, or whether they can be understood within conventional perturbative dynamics. To this effect we have used the latest available technology to describe charm production over the entire range of $x$ and $Q^{2}$ accessible to experiment. Our approach consistently interpolates between the two asymptotic regions of massless evolution at large $Q^{2}$ and the photon-gluon fusion process at low $Q^{2}$, and includes quark and target mass effects and corrections for mass thresholds.

To a certain extent our findings confirm the existing state of affairs, in which some of the data show no evidence at all for intrinsic charm, while other data cannot be fitted without additional non-perturbative input. Even within the rather different models of intrinsic charm considered here, with varying normalizations, it seems difficult to simultaneously fit the entire data set in terms of a single intrinsic charm scenario - although there may be a slight preference for intrinsic charm in model IC2 at a level of about $0.4 \%$. The clearest conclusion that one can draw from this is that more quality data are urgently needed to settle the issue. In particular, while the small- $x$ domain seems to be relatively well under control, the large- $x$ region, where cross sections are small and measurements more difficult, must be the focus of future experimental effort if the non-perturbative structure of the nucleon sea is to be resolved.

\section{ACKNOWLEDGMENTS}

W.M. and F.M.S. would like to acknowledge the support of the Special Research Centre for the Subatomic Structure of Matter at the University of Adelaide during the initial stages of this work. F.M.S. would like to thank S.J. Brodsky, S. Kretzer and F.S. Navarra for

discussions. This work was supported by the Australian Research Council and by FAPESP (Brazil). 


\section{REFERENCES}

[1] H1 Collaboration, C. Adloff et al., Z. Phys. C 72 (1996) 593.

[2] ZEUS Collaboration, J. Breitweg et al., Phys. Lett. B 407 (1997) 402.

[3] European Muon Collaboration, J.J. Aubert et al., Nucl. Phys. B213 (1983) 31; Phys. Lett. B 94 (1980) 96; ibid. 110 B (1982) 73.

[4] S.J. Brodsky, P. Hoyer, C. Peterson and N. Sakai, Phys. Lett. 93 B (1980) 451; S.J. Brodsky, C. Peterson and N. Sakai, Phys. Rev. D 23 (1981) 2745.

[5] E. Hoffman and R. Moore, Z. Phys. C 20 (1983) 71.

[6] B.W. Harris, J. Smith and R. Vogt, Nucl. Phys. B461 (1996) 181.

[7] G. Ingelman and M. Thunman, Z. Phys. C 73 (1997) 505.

[8] Yu.A. Golubkov, hep-ph/9811218.

[9] R. Vogt and S.J. Brodsky, Phys. Lett. B 349 (1995) 569; R. Vogt, Nucl. Phys. B446 (1995) 149.

[10] J. Badier et al., Phys. Lett. 114 B (1982) 457; ibid. 158 B (1985) 85.

[11] C. Biino et al., Phys. Rev. Lett. 58 (1987) 2523.

[12] A.D. Martin, R.G. Roberts, W.J. Stirling and R.S. Thorne, Eur. Phys. J. C 4 (1998) 463.

[13] M. Glück, E. Reya and A. Vogt, Eur. Phys. J. C 5 (1998) 461.

[14] M.A.G. Aivazis, J.C. Collins, F.I. Olness and W.-K. Tung, Phys. Rev. D 50 (1994) 3102.

[15] S. Kretzer and I. Schienbein, Phys. Rev. D 58 (1998) 094035.

[16] J.C. Collins and W.-K. Tung, Nucl. Phys. B278 (1986) 934;

[17] CTEQ Collaboration, R. Brock et al., Handbook of Perturbative QCD, ed. G. Sterman, Fermilab-Pub-93/094.

[18] M. Buza, Y. Matiounine, J. Smith and W.L. van Neerven, Eur. Phys. J. C 1 (1998) 301.

[19] B.W. Harris and J. Smith, Nucl. Phys. B452 (1995) 109.

[20] A.D. Martin, R.G. Roberts, M.G. Ryskin and W.J. Stirling, Eur. Phys. J. C 2 (1998) 287.

[21] R. S. Thorne and R. G. Roberts, Phys. Rev. D 57 (1998) 6871; Phys. Lett. B 421 (1998) 303.

[22] F.M. Steffens, Nucl. Phys. B523 (1998) 487.

[23] D. Drijard et al., Phys. Lett. 81 B (1979) 250; ibid. 85 B (1979) 452; K.L. Giboni et al., ibid. 85 B (1979) 437; W. Lockman et al., ibid. 85 B (1979) 443; A. Chilingarov et al., ibid. 83 B (1979) 136; H1 Collaboration, S. Aid et al., Phys. Lett. B 379 (1996) 319.

[24] R. Vogt and S.J. Brodsky, Nucl. Phys. B438 (1995) 261.

[25] F.S. Navarra, M. Nielsen, C.A.A. Nunes and M. Teixeira, Phys. Rev. D 54 (1996) 842.

[26] S. Paiva, M. Nielsen, F.S. Navarra, F.O. Duraes and L.L. Barz, Mod. Phys. Lett. A 13 (1998) 2715.

[27] W. Melnitchouk and A.W. Thomas, Phys. Lett. B 414 (1997) 134.

[28] H1 Collaboration, C. Adloff et al., Z. Phys. C 74 (1997) 191; ZEUS Collaboration, J. Breitweg et al., Z. Phys. C 74 (1997) 207.

[29] J.F. Gunion and R. Vogt, University of California-Davis Report UCD-97-14, LBNL40399 [hep-ph/9706252].

[30] New Muon Collaboration, P. Amandruz et al., Phys. Rev. Lett. 66 (1993) 2712; NA51 Collaboration, A. Baldit et al., Phys. Lett. B 332 (1994) 244; E866/NuSea Collaboration, E.A. Hawker et al., Phys. Rev. Lett. 80 (1998) 3715. 
[31] W. Melnitchouk, J. Speth and A.W. Thomas, Phys. Rev. D 59 (1998) 014033; J. Speth and A.W. Thomas, Adv. Nucl. Phys. 24 (1998) 83; A.W. Thomas, Phys. Lett. B 126 (1983) 97.

[32] A.I. Signal and A.W. Thomas, Phys. Lett. 191 B (1987) 205; X. Ji and J. Tang, Phys. Lett. B 362 (1995) 182; M.J. Musolf et al., Phys. Rep. 239 (1994) 1; W. Melnitchouk and M. Malheiro, Phys. Rev. C 55 (1997) 431.

[33] F.S. Navarra and M. Nielsen, Phys. Lett. B 443 (1998) 285.

[34] B. Mueller et al., Phys. Rev. Lett. 78 (1997) 3824; K.A. Aniol et al., Phys. Rev. Lett. 82 (1999) 1096.

[35] CTEQ Collaboration, H.L. Lai, J. Huston, S. Kuhlmann, J. Morfin, F. Olness, J.F. Owens, J. Pumplin and W.-K. Tung, Michigan State U. report number MSUHEP/903100, hep-ph/9903282. 


\section{FIGURES}

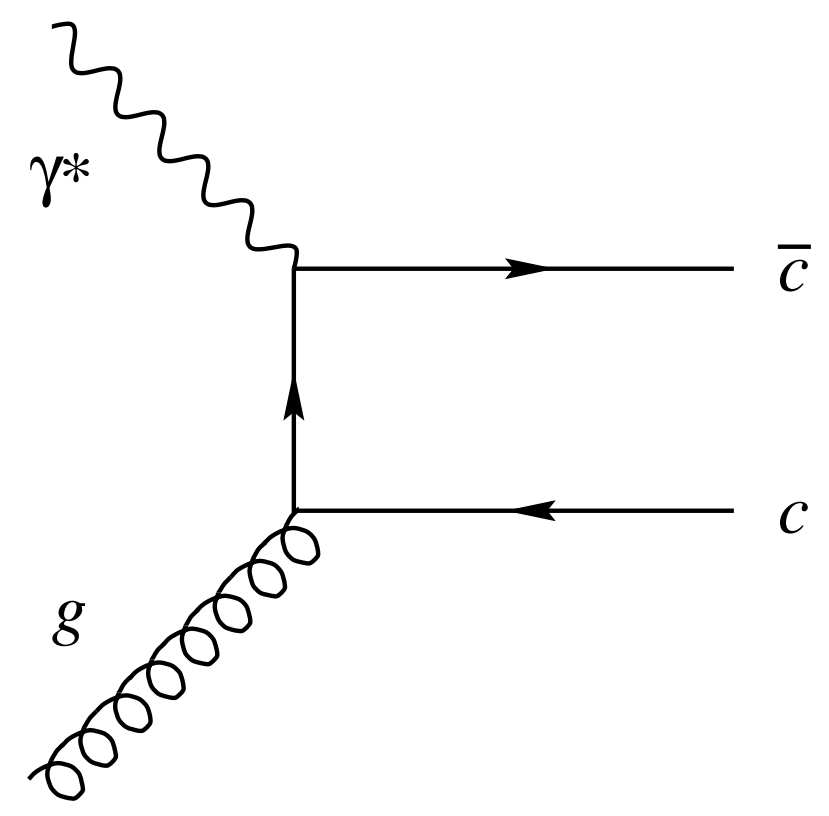

FIG. 1. Photon-gluon fusion process at leading order in $\alpha_{s}$. 

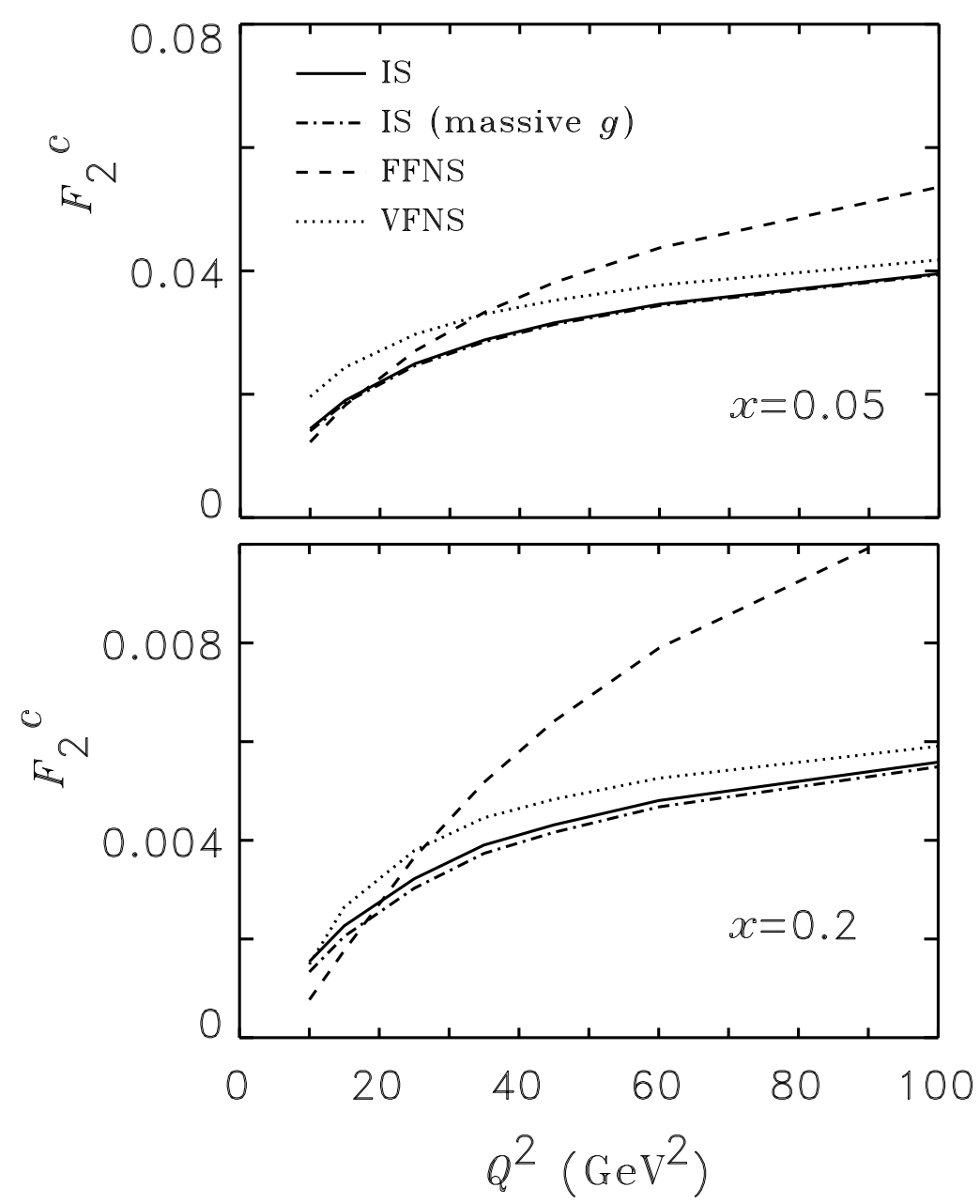

FIG. 2. Charm structure function as a function of $Q^{2}$ for $x=0.05$ and 0.2 , evaluated according to the various schemes discussed in the text. "IS" refers to the interpolating scheme of Eq.(9), while "IS (massive $g$ )" denotes the interpolating scheme but with massless quark coefficient functions. The GRV parameterization [13] for the gluon is used. 

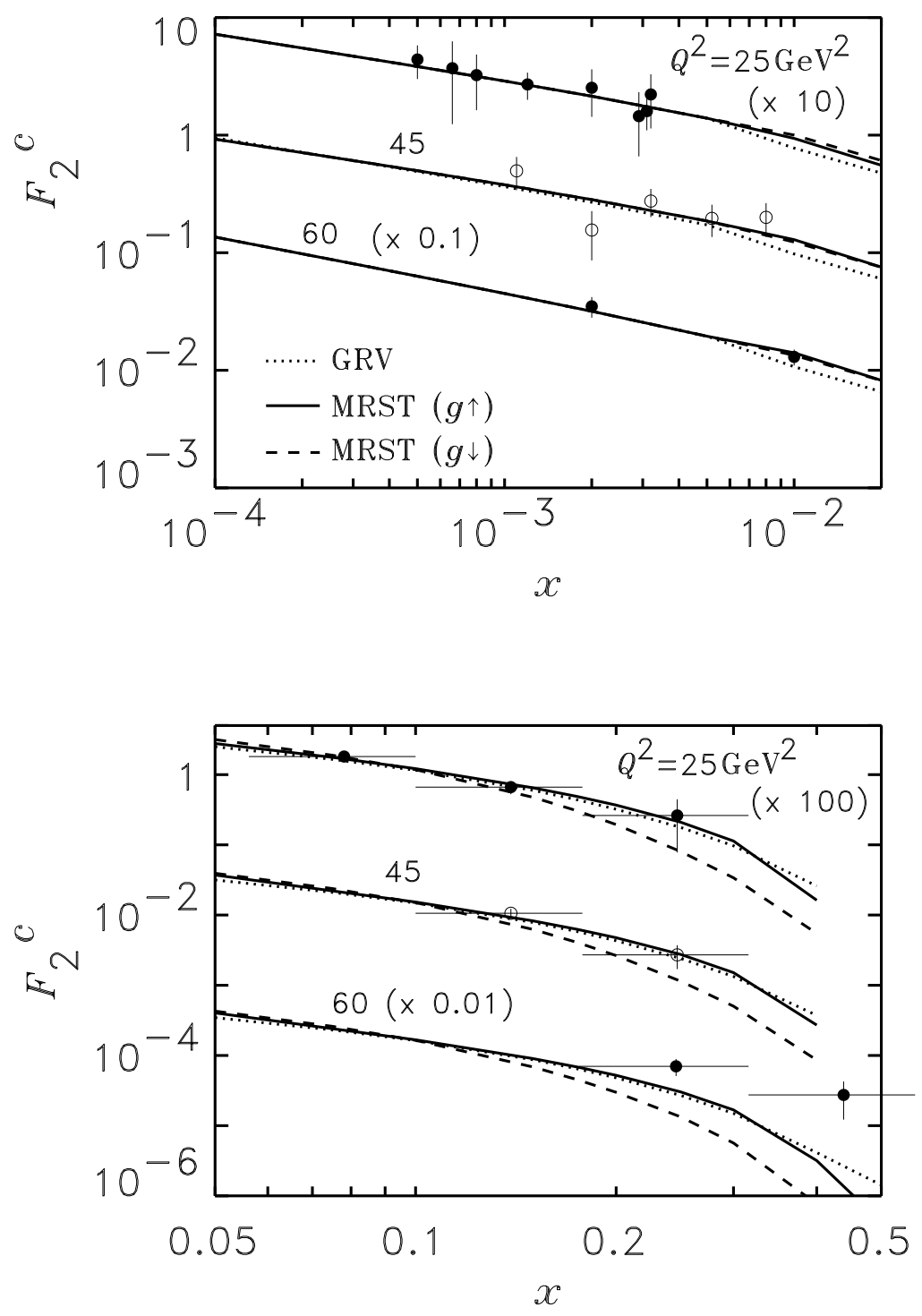

FIG. 3. Charm structure function calculated within the interpolating scheme for different gluon distributions, GRV [13] (dotted) and MRST [12] with minimum (dashed) and maximum (solid) gluons. The data at small $x$ are from the ZEUS Collaboration [2], while the large- $x$ data are from the EMC [3]. For clarity the small- $x$ curves have been scaled by a factor $10(0.1)$ for $Q^{2}=25(60)$ $\mathrm{GeV}^{2}$, and the large- $x$ curves by a factor $100(0.01)$. 


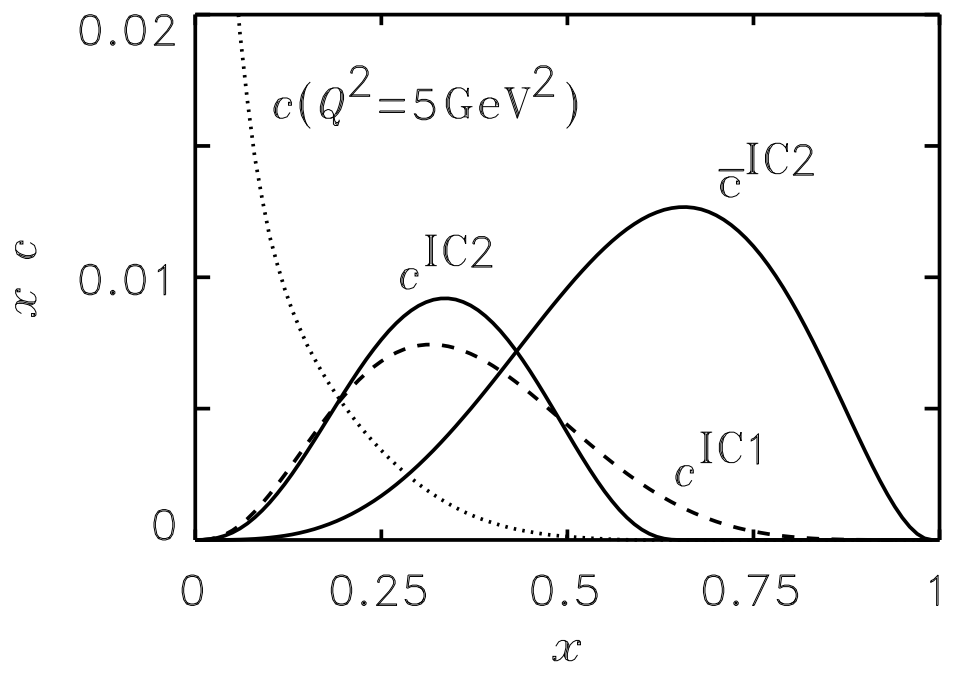

FIG. 4. Charm quark distributions from the intrinsic charm models IC1 27 (solid) and IC2 [4] (dashed), both normalized to $1 \%$, and from the MRST parameterization [12] (with maximal gluon) at $Q^{2}=5 \mathrm{GeV}^{2}$ (dotted). 


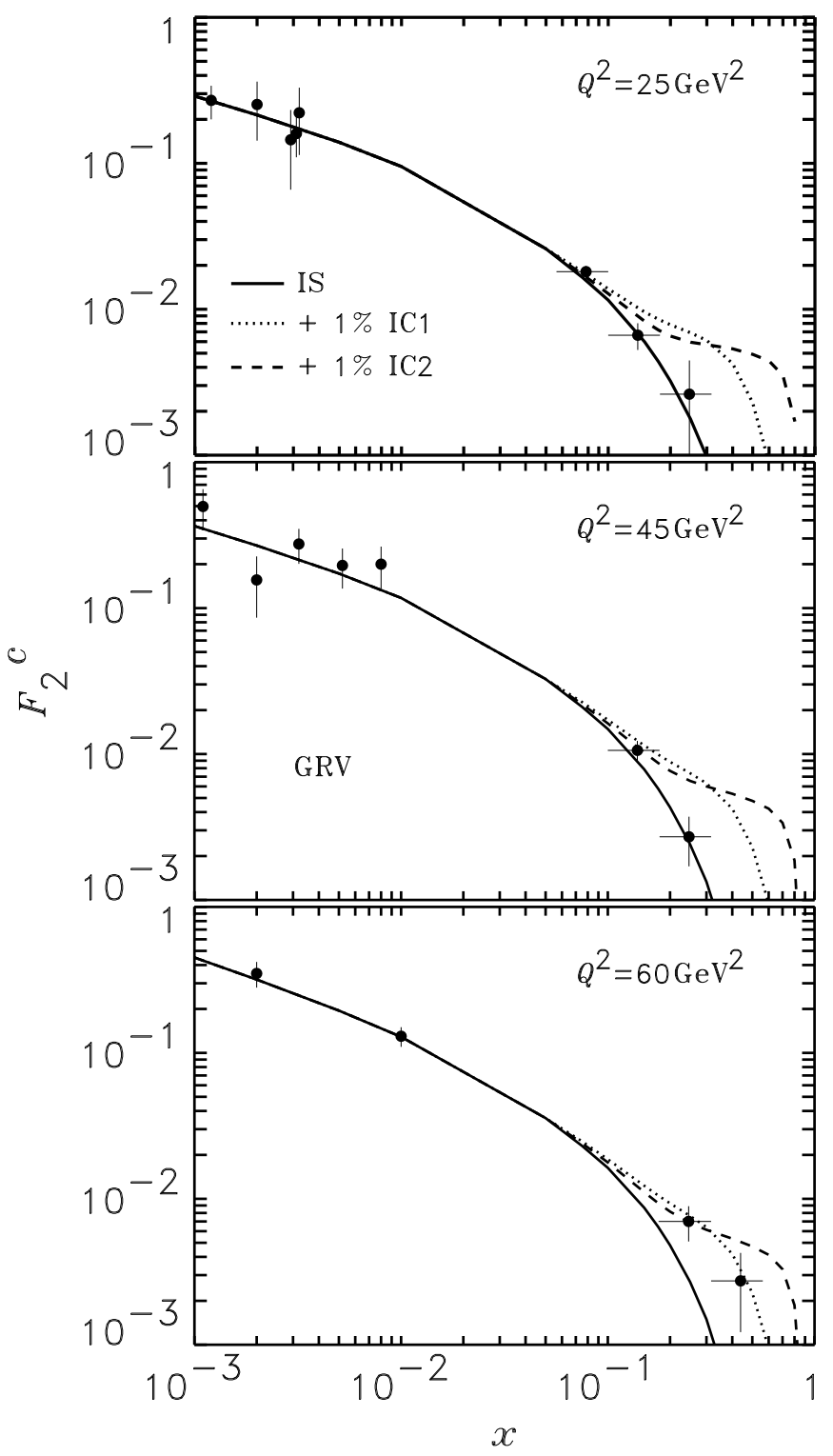

FIG. 5. The charm structure function calculated through the interpolating scheme ("IS") in Eq.(9) for intrinsic charm distributions from models IC1 and IC2, normalized to 1\%. The GRV parameterizations [13] for the gluon and light quark densities are used. 


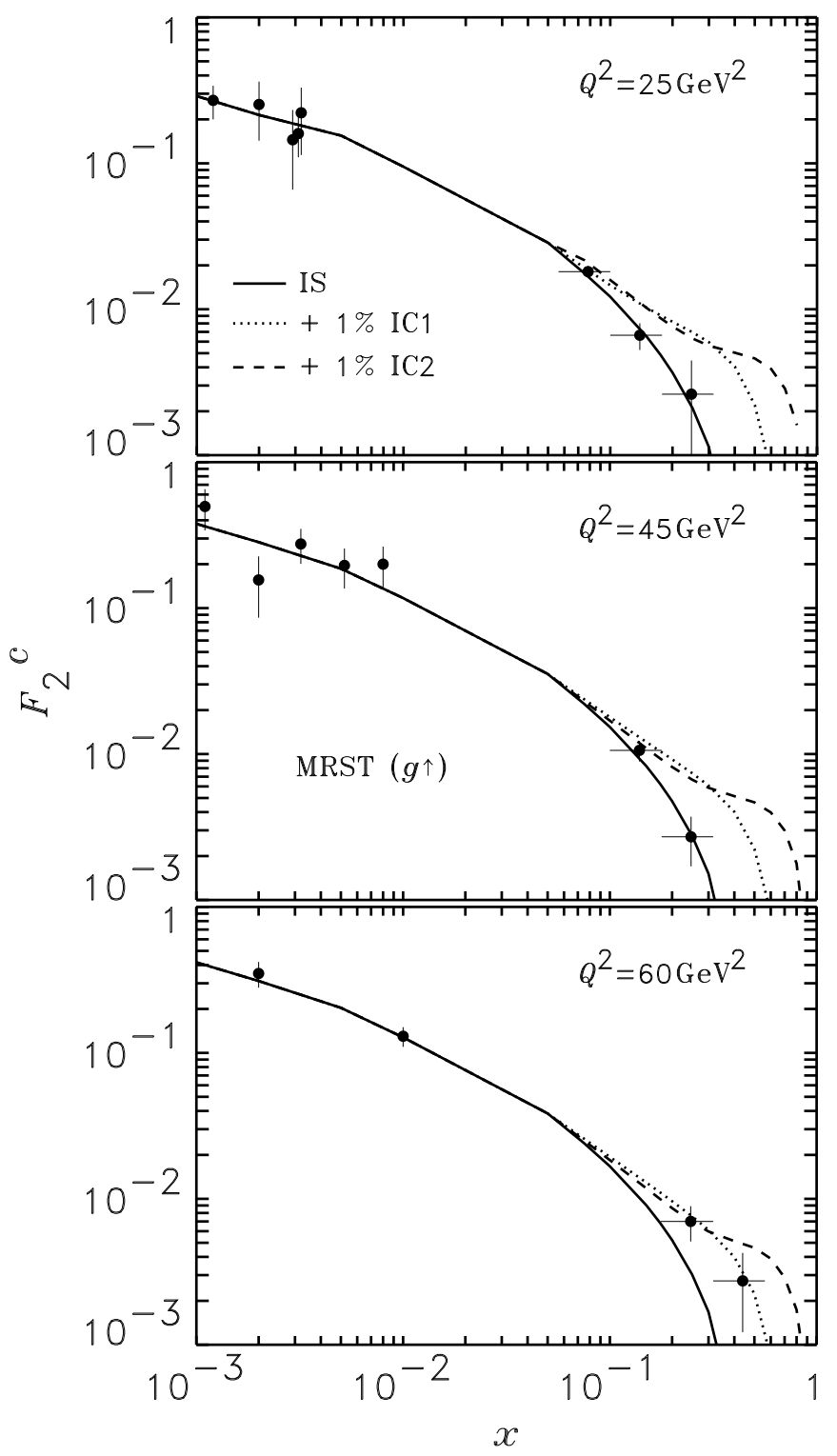

FIG. 6. As in Figure 5, but with the MRST parameterization 12] with the maximum gluon. 


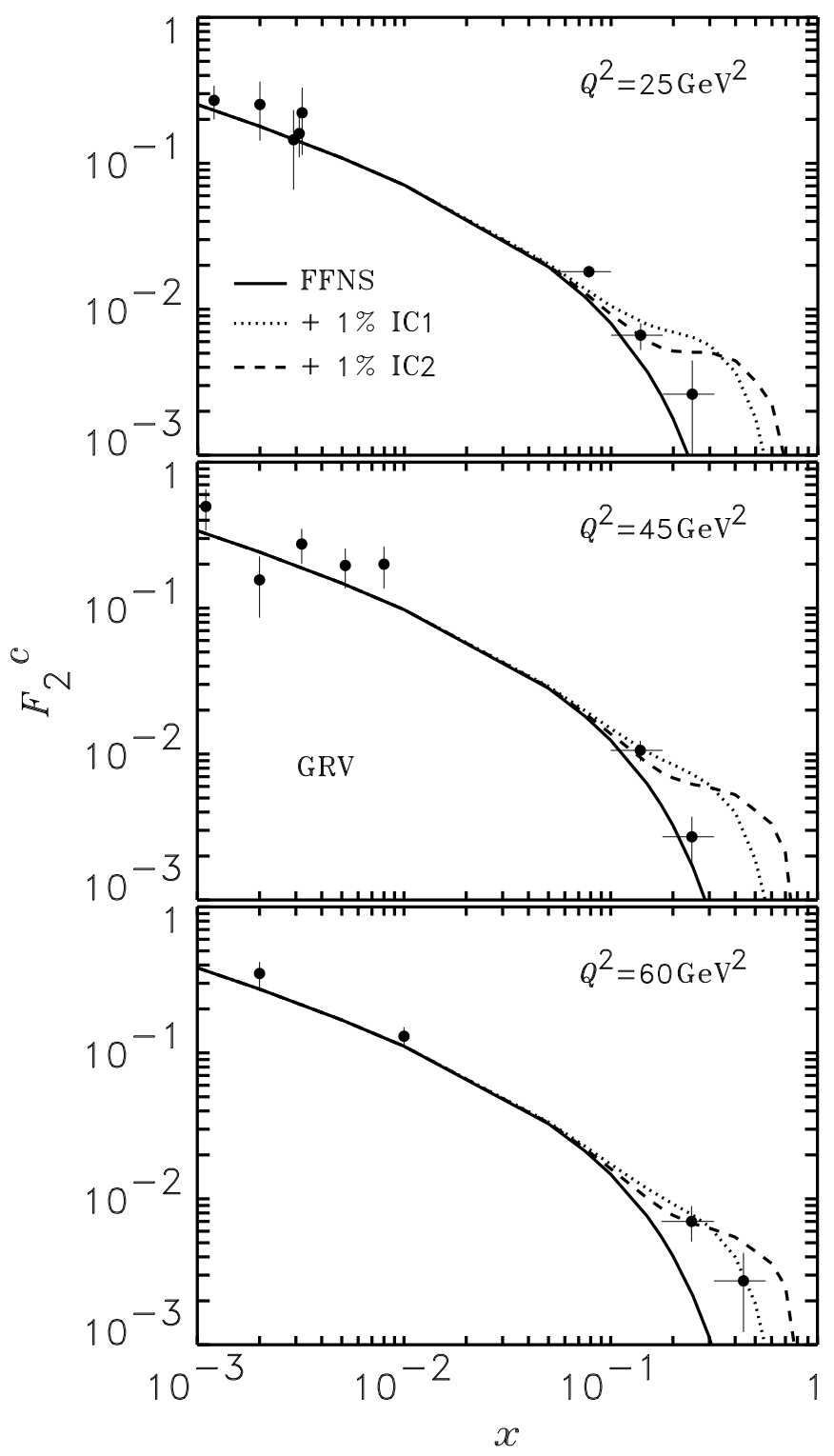

FIG. 7. As in Figure 5, but with $F_{2}^{c}$ calculated according to the FFNS in Eq.(11). 

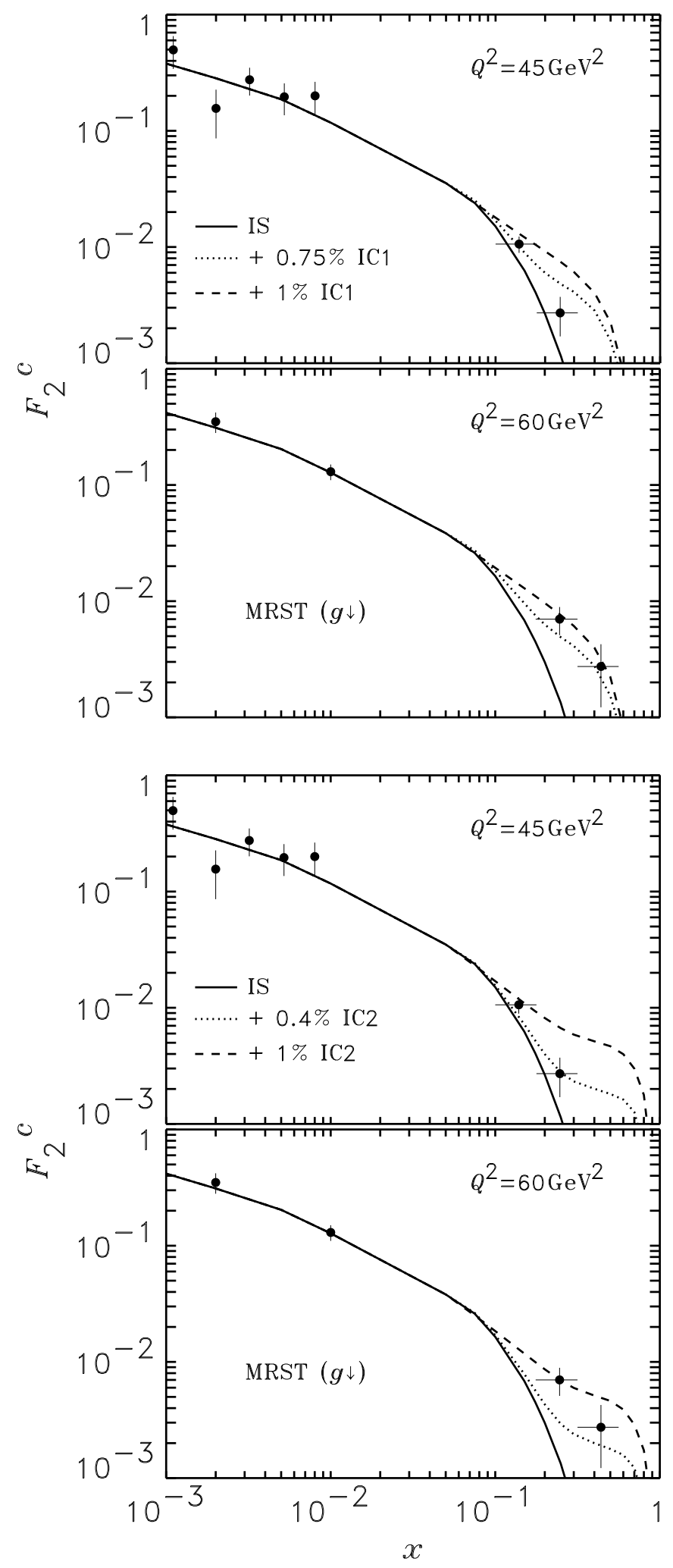

FIG. 8. As in Figure 5, but with different normalizations for the IC1 and IC2 model distributions, and with the MRST parameterization [12] with the minimum gluon. 


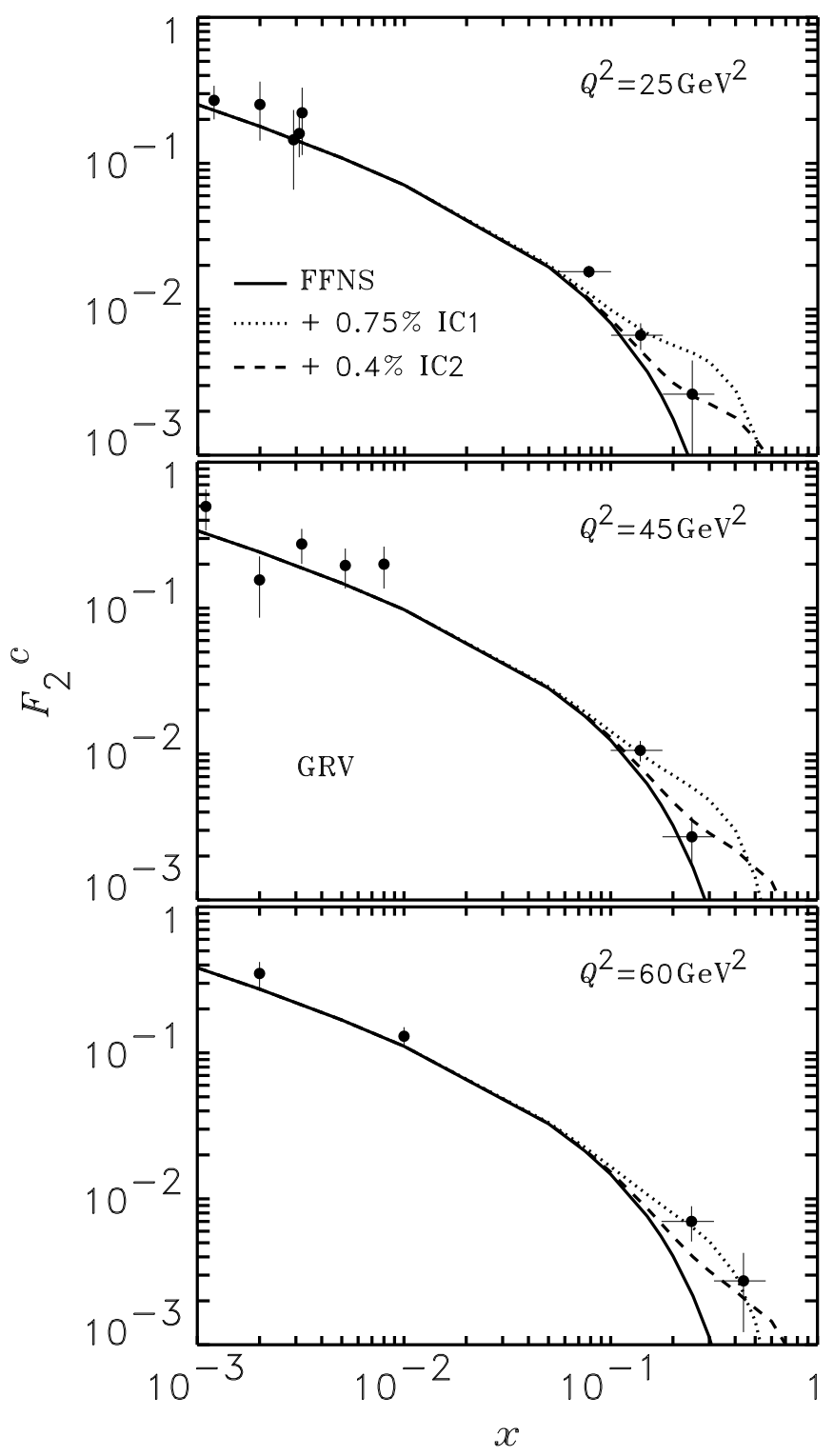

FIG. 9. As in Figure 1, but with different normalizations for the IC1 and IC2 components. 